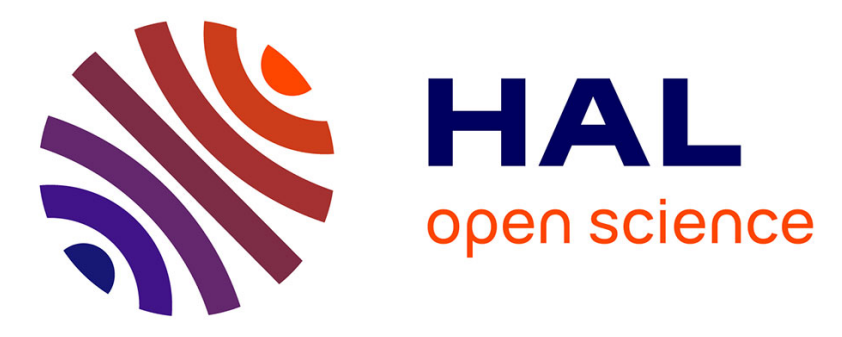

\title{
Slip dynamics at a patterned rubber/glass interface during stick-slip motions
}

M.C. Audry, Christian Frétigny, A. Chateauminois, J. Teisseire, E. Barthel

\section{To cite this version:}

M.C. Audry, Christian Frétigny, A. Chateauminois, J. Teisseire, E. Barthel. Slip dynamics at a patterned rubber/glass interface during stick-slip motions. European Physical Journal E: Soft matter and biological physics, 2012, 35, pp.83. 10.1140/epje/i2012-12083-0 . hal-00731424

\section{HAL Id: hal-00731424 \\ https://hal.science/hal-00731424}

Submitted on 12 Sep 2012

HAL is a multi-disciplinary open access archive for the deposit and dissemination of scientific research documents, whether they are published or not. The documents may come from teaching and research institutions in France or abroad, or from public or private research centers.
L'archive ouverte pluridisciplinaire HAL, est destinée au dépôt et à la diffusion de documents scientifiques de niveau recherche, publiés ou non, émanant des établissements d'enseignement et de recherche français ou étrangers, des laboratoires publics ou privés. 


\title{
Slip dynamics at a patterned rubber/glass interface during stick-slip motions
}

\author{
M.C Audry ${ }^{1}$, C. Fretigny ${ }^{1}$, A. Chateauminois ${ }^{1}$, J. Teissere $^{2}$, and E. Barthel ${ }^{2}$ \\ ${ }^{1}$ Laboratoire de Physico-Chimie des Polymères et des Milieux Dispersés, UMR CNRS 7615, Ecole Supérieure de Physique et \\ Chimie Industrielles (ESPCI), Université Pierre et Marie Curie, Paris, France. \\ 2 Surface du Verre et Interfaces, UMR 125, CNRS/ Saint Gobain, Aubervilliers, France
}

Received: / Revised version:

\begin{abstract}
We report on an experimental study of heterogeneous slip instabilities generated during stickslip motions at a contact interface between a smooth rubber substrate and a patterned glass lens. Using a sol-gel process, the glass lens is patterned with a lattice of parallel ridges (wave length, $1.6 \mu \mathrm{m}$, amplitude $0.35 \mu \mathrm{m}$ ). Friction experiments using this patterned surface result in the systematic occurrence of stick-slip motions over three orders of magnitude in the imposed driving velocity while stable friction is achieved with a smooth surface. Using a contact imaging method, real-time displacement fields are measured at the surface of the rubber substrate. Stick-slip motions are found to involve the localized propagation of transverse interface shear cracks whose velocity is observed to be remarkably independent on the driving velocity.
\end{abstract}

PACS. $46.50+\mathrm{d}$ Tribology and Mechanical contacts - 62.20 Qp Friction, Tribology and Hardness

\section{Introduction}

Stick-slip oscillations are observed in many natural and man-made mechanical systems such as brakes [1], granular materials under shear $[2,3]$ or the bowing of a violin string [4], to cite a few example. From a macroscopic point of view, these processes are often described by considering that frictional instabilities involve the more or less periodic jump from a purely elastic state without any slip to a homogeneous sliding state. Within the framework of Amonton-Coulomb's law, static and dynamic coefficient of friction concepts are often ascribed to these processes. Many theoretical works have been devoted to stick-slip motions, including the seminal 'rate and state' model proposed by Rice and Ruina $[5,6]$ which can satisfactorily reproduce the different regimes of frictional sliding and the bifurcations between them. One of the inherent limitation of such descriptions is that they usually consider the sliding motions between perfectly rigid bodies. As a consequence, any spatial inhomogeneity in the frictional process at the contact interface is discarded. However, many theoretical works have postulated $[7-10]$ that the nucleation of frictional sliding between deformable bodies does not necessarily occurs homogeneously. Then, sliding is assumed to occur as a result of rapid fracture-like modes at the contact interface.

Although scarce, experimental evidence of such crack-like processes emerged from recent studies using the resources

Send offprint requests to: antoine.chateauminois@espci.fr of real time contact imaging. In a series of works using extended contact interfaces between rough PMMA blocks, Rubinstein and co-corkers [11-14] have especially observed that upon the application of shear the onset of sliding motions is preceded by a discrete sequence of crack-like precursors which are initiated at shear levels that are well below the threshold for static friction. In these studies, the location and the velocity of the crack fronts were determined from the associated redistribution of contact points within the multi-contact interface. Few cases of similar inhomogeneous mode of sliding have been reported in laboratory experiments on soft systems such as polyurethane over Araldite [15] and gelatin gels over glass [16]. In a detailed study with a gelatin block sliding over a glass substrate, Ronsin and co-workers [17-19] have observed that the nucleation of frictional sliding under constant stress loading involves the stationary propagation of localized sliding zones denoted to as "self-healing pulses" which are nucleated either at the edges of the contact or inside it. Noticeably, these pulses are different from the extensively studied Schallamach waves instabilities [20] in the sense that they do not involve any detectable detachment of the contacting surfaces. Thus, they involve a complex and poorly understood interplay between the local friction law and the fracture mechanics of the pulse. More generally, the experimental determination of the relevant system properties (contact geometry, loading conditions, material and surface properties) for the occurrence of crack-like instabilities at frictional interfaces remains 
an open issue

In this study, we report on new observations of cracklike processes at a frictional interface between a rubber substrate and a glass lens patterned with sub-micrometer ridges. Under displacement driven conditions, patterning the glass surface results in the systematic occurrence of stick-slip processes over three order of magnitude in the imposed velocity. The dynamics of the associated slip processes is further investigated using a previously developed contact imaging method which allows to measure spatially resolved sliding velocity fields [21-23] within the contact interface.

\section{Experimental details}

Frictional experiments are carried out within a contact between a smooth silicone rubber substrate and a glass lens patterned with micrometer sized ridges. A commercially available transparent Poly(DiMethylSiloxane) (PDMS) silicone (Sylgard 184, Dow Corning, Midland, MI) is used as an elastomer substrate. In order to monitor contact induced surface displacements, a square network of small cylindrical holes (diameter $8 \mu \mathrm{m}$, depth $11 \mu \mathrm{m}$ and centerto-center spacing $100 \mu \mathrm{m}$ ) is produced on the PDMS surface by means of conventional micro-lithography techniques. Under transmitted light observation conditions, this pattern appears as a network of dark spots which are easily detected using image processing. Full details regarding the elaboration of PDMS substrate are given in $[22,23]$. The Young's modulus of the PDMS substrate is 3.15 MPa. Specimen size $\left(15 \times 60 \times 60 \mathrm{~mm}^{3}\right)$ ensures that semi infinite contact conditions are achieved during friction experiments (i.e. the ratio of the substrate thickness to the contact radius $(a=2.5 \mathrm{~mm})$ is greater than ten [24]). Before use, PDMS specimens are thoroughly washed with isopropanol and subsequently dried under vacuum. Contacts are achieved between the PDMS substrate and a patterned plano-convex glass lens (Melles Griott, France) with a radius of curvature of $25 \mathrm{~mm}$. A ridge pattern is realized at the surface of the glass lens using previously developed embossing techniques [25]. A reactive sol-gel solution is spin coated onto the glass lens and subsequently squeezed by a PDMS template with a negative imprint of the desired morphology on its surface. After curing 2 hours at $90^{\circ} \mathrm{C}$ and demolding, a regular ridge pattern is obtained as shown in Figure 1. Atomic Force Microscopy (AFM) measurements indicates that the height of the ridge is $350 \mathrm{~nm}$ and that the wave length is $1.6 \mu \mathrm{m}$.

Friction experiments are performed under imposed normal load $(1.45 \mathrm{~N})$ and velocity (from $5 \mu \mathrm{m} \mathrm{s}^{-1}$ to $2 \mathrm{~mm} \mathrm{~s}^{-1}$ ) using a home made device already used in former studies $[21,23]$. The apparatus is based on a combination of leaf springs loaded in tensile mode along the vertical and lateral directions in order to ensure a high stiffness (> $510^{5} \mathrm{~N} \mathrm{~m}^{-1}$ ) along these two directions while preserving a good sensitivity in the friction force measurement. The PDMS substrate is displaced with respect to the fixed glass lens by means of a linear translation stage. In all the experiments to be reported, the ridges of the patterned
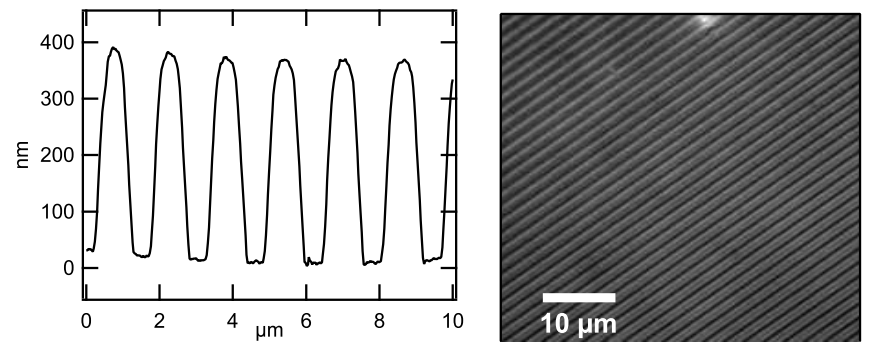

Fig. 1. Topography of the ridge pattern on the glass lens. Right: optical microscope picture; left: AFM trace taken perpendicular to ridge orientation.

glass lens are oriented perpendicularly to the sliding direction. Lateral load and displacement are continuously recorded by means of a piezzoelectric load cell (Kistler, Germany) and a laser transducer (Philtec, USA), respectively. It is worth noting that the lateral stiffness of the device is about 50 times higher than the lateral contact stiffness. As a consequence, the observed stick-slip motions do not incorporate any significant inertia coupling with the friction device, as it is often the case with conventional friction set-ups based on compliant leaf springs arrangements. During sliding, images of the deformed contact zone are continuously recorded through the transparent PDMS substrate up to a rate of 130 frames per second using a zoom lens and a CMOS camera. The integration time of the CMOS sensor is set to $0.5 \mathrm{~ms}$. The system is configured to a frame size of $(1024 \times 1024)$ pixels with 8 bits resolution. It should be mentioned that the stick-slip processes to be reported are not associated with any evidence of localized detachment waves such as Shallamach waves [20]. Within the limits of the optical resolution of the optical device (a few $\mu \mathrm{m}$ ), it was indeed not possible to detect any detachment of the contacting surfaces during slipping phases.

\section{Results}

Figure 2 shows the friction traces obtained when the imposed velocity is increased by about three decades, from $5 \mu \mathrm{m} \mathrm{s}^{-1}$ to $2 \mathrm{~mm} \mathrm{~s}^{-1}$. Stick-slip events are evidenced within the whole investigated velocity range although the amplitude of the force fluctuations is vanishing in the $\mathrm{mm} \mathrm{s}^{-1}$ range. On the other hand, stable sliding is achieved at all velocities if a glass lens with a smooth sol-gel coating is used instead of the patterned one. Stick-slip motions are thus induced by the presence of the ridge pattern. A potential explanation for this phenomenon could be that the contact interface is weakened by localized stress fluctuations induced at the surface of the PDMS substrate by the micrometer sized ridges. As shown in Figure 2, the shape of the friction traces is evolving as a function of the driving velocity. At low velocity $\left(5 \mu \mathrm{m} s^{-1}\right.$, figure $\left.2 \mathrm{a}\right)$, a very regular saw tooth signal is obtained where nearly linear rises in the lateral force alternate with sudden load 

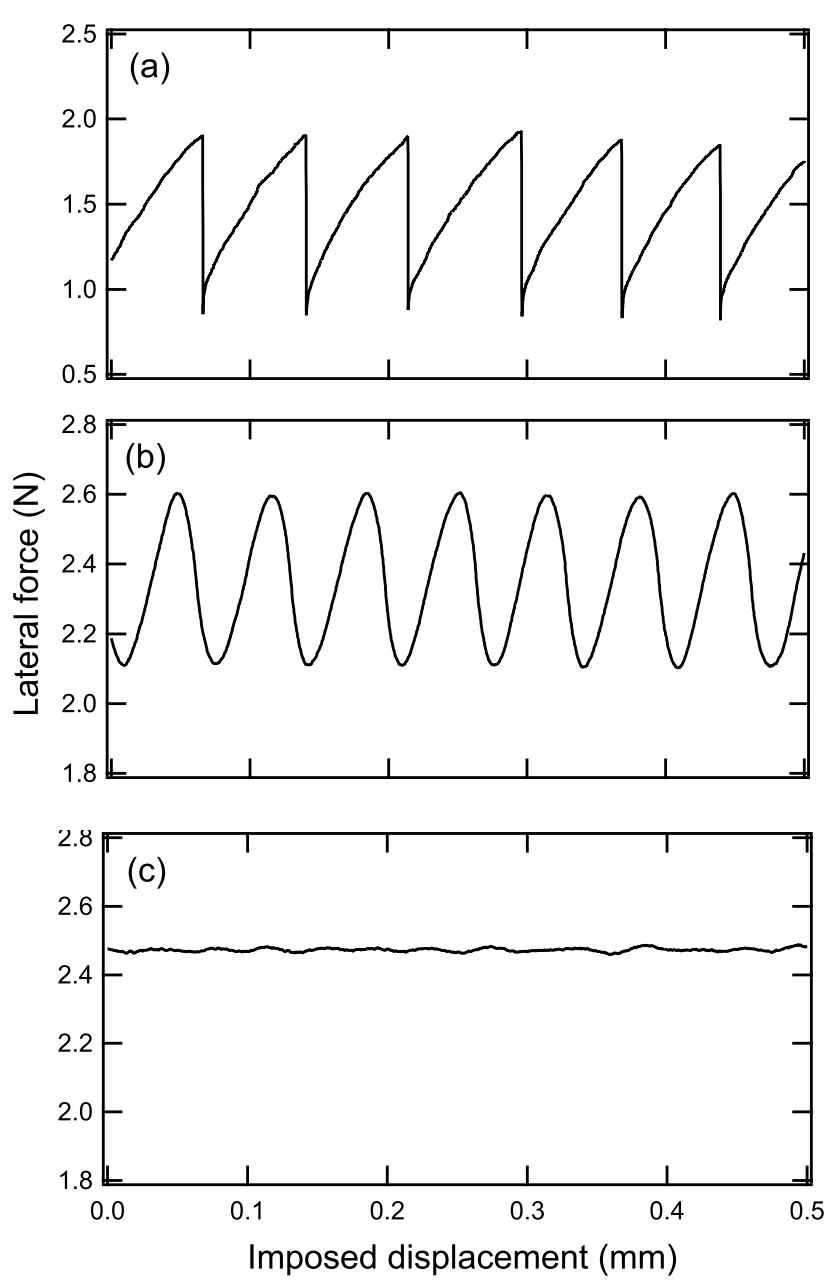

Fig. 2. Friction force traces as a function of the sliding velocity. (a) $5 \mu \mathrm{m} \mathrm{s}^{-1}$, (b) $1 \mathrm{~mm} \mathrm{~s}^{-1}$, (c) $2 \mathrm{~mm} \mathrm{~s}^{-1}$.

drops. Such a response is reminiscent of the usual description of stick-slip motions as the sharp transition from an elastic 'stick' state to a frictional 'slip' state when the socalled static friction threshold is exceeded. On the other hand, a load response intermediate between linear and sinusoidal is observed at higher velocity $\left(1 \mathrm{~mm} \mathrm{~s}^{-1}\right.$, figure $2 \mathrm{~b}$ ) with no clear evidence of a stick phase. As compared to low velocity, the amplitude of the load fluctuations is also strongly reduced (figure 3 ). At $2 \mathrm{~mm} \mathrm{~s}^{-1}$, low amplitude but distinguishable load fluctuations are still evidenced in the friction trace (figure 2c). Over the whole investigated velocity range, the average stick-slip frequency, $F$, is nearly linearly related to the driving velocity, $v$, (figure 4) independently of the details of the friction trace. A characteristic length of the order of $70 \mu \mathrm{m}$ is obtained from the ratio $v / F$. Incidentally, this length is very close to the spacing $(100 \mu \mathrm{m})$ between markers at the surface of the PDMS substrate. However, it can be seen from the data reported in figure 2 that identical stick-slip frequencies are obtained with an unmarked PDMS surface. The characteristic length extracted from the frequency-velocity relationship is thus not correlated with the presence of the

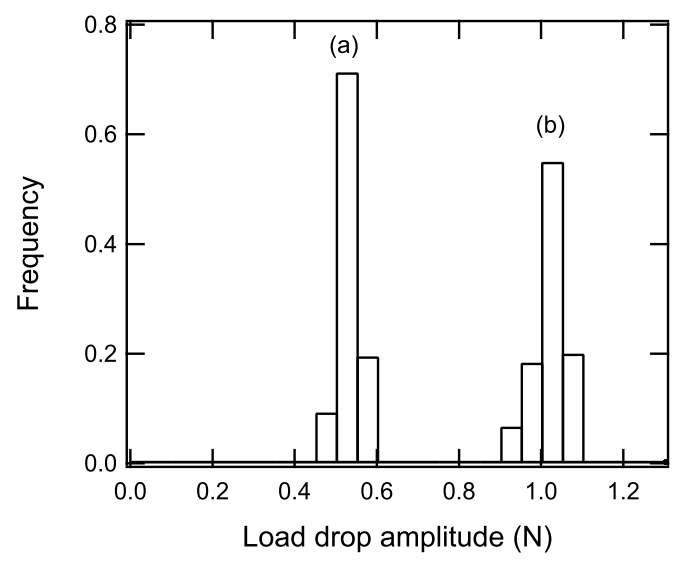

Fig. 3. Histograms giving the distribution of the load drops associated with stick-slip events. (a) $1 \mathrm{~mm} \mathrm{~s}^{-1}$, (b) $5 \mu \mathrm{m} \mathrm{s}^{-1}$.

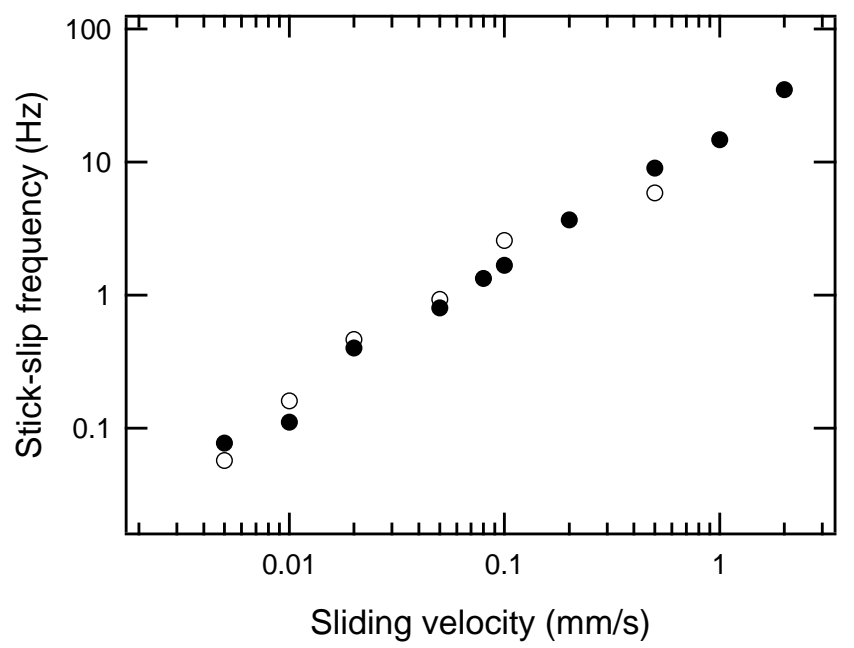

Fig. 4. Stick-slip frequency as a function of the imposed sliding velocity. $(\bullet)$ marked PDMS substrate; (०) unmarked PDMS substrate

holes lattice at the surface of the PDMS.

Figure 5 details the friction force trace during a stick phase at $5 \mu \mathrm{m} \mathrm{s}^{-1}$. Immediately after the occurrence of a load drop event, a sharp negative peak is observed with a characteristic time of the order of one second. This phenomena is probably related to a viscoelastic load retardation effect associated with the rapid displacement of the PDMS surface during the slip event. After the occurrence of this peak, a nearly linear load response is observed with a slope close to that corresponding to the lateral contact stiffness (as indicated by the dotted line in the figure). Here, the lateral contact stiffness, $K=$ $d F_{t} / d \delta=1.410^{4} \mathrm{~N} \mathrm{~m}^{-1}$, (where $F_{t}$ is the lateral force and $\delta$ the imposed displacement) is measured independently during the incipient stages of the stiction process, i.e. when the contact is sheared without any detectable slip at the interface. As expected, this experimental value of the lateral stiffness is very close to the theoretical prediction corresponding to the drag of a rigid disk at the surface of an incompressible semi-infinite elastic media 

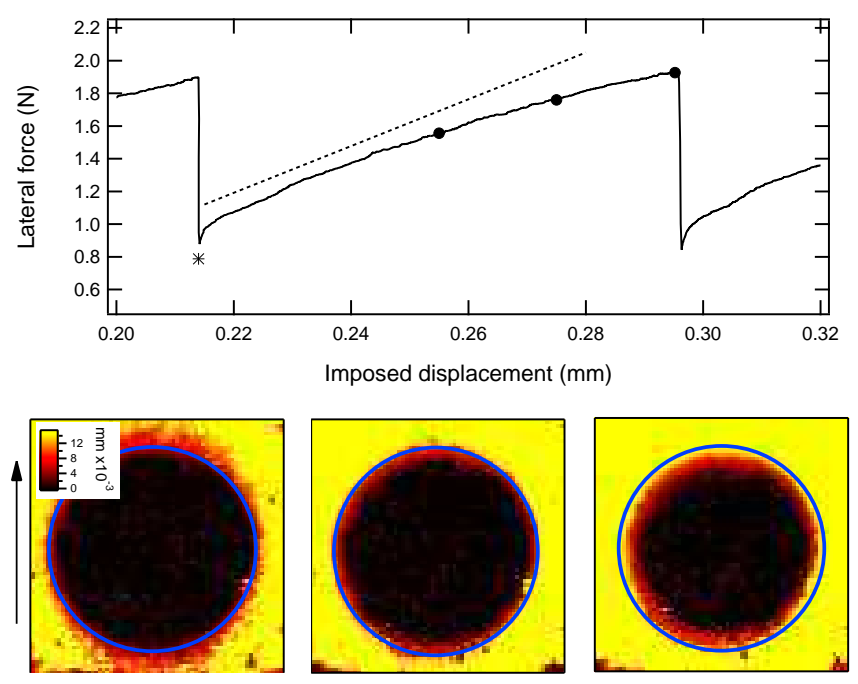

Fig. 5. Surface displacement field during the stick phase at $5 \mu \mathrm{m} \mathrm{s}^{-1}$. Top: friction trace. The dotted line corresponds to the contact stiffness as it is measured independently during incipient sliding (see text for details). Bottom: surface displacement fields recorded at increasing times (from left to right) during the stick phase. The corresponding loads and displacements are indicated by filled circles on the friction trace. The image just following the slip event marked by an asterix was taken as a reference for the measurement of surface displacements. As indicated by the vertical arrow, the PDMS substrate is moved from bottom to top with respect to the contact area which is delimited by a blue circle. Some slip is seen to occur at the periphery of the contact when the load is increased. The contact radius is $2.5 \mathrm{~mm}$.

(i.e. $K=16 / 3 G a$, where $G$ is the shear modulus and $a$ is the contact radius) [26]. When the lateral load is further increased up to the threshold corresponding to the occurrence of a slip event, some negative deviation from this purely elastic contact response is observed. As shown by the surface displacement fields in the bottom part of figure (5), the observed non linearity is associated with some localized slip propagation within an annular ring at the periphery of the contact. Similar slip processes have already been reported during the quasi-static stiction of contacts between smooth glass spheres and rubbers [27, 22 , where they are accounted for by stress concentration at the periphery of the adhesive contact $[28,29]$. Here, it is clear that the so-called 'stick phase' in fact involves some localized slip within the contact interface. Figure 6 provides additional details regarding surface displacements during a slip event at the same imposed velocity $\left(5 \mu \mathrm{m} \mathrm{s}^{-1}\right)$. From the monitoring of marker's location at the surface of the PDMS substrate, the displacement component along the sliding direction is measured at regular time increments (the reference image for the measurement of the displacements is taken immediately after the occurrence of the previous slip event). Then, the time derivative of this component provides the actual sliding velocity at the contact interface. When looking at the sliding velocity fields shown in the top part of the figure, it comes out that the slip phase involves the propagation of a straight, localized, slip pulse - or interface crack- across the contact. Such pulses are systematically initiated at one of the contact lateral edges (left or right) and propagate in a direction perpendicular to the imposed sliding velocity. The bottom part of the figure provides the corresponding displacement profiles across the contact zone at regular time intervals (the profiles are taken along a direction perpendicular to the sliding direction). The two first profiles in the bottom part of the figure correspond to the displacements just before the nucleation of the interface crack. As detailed above, some localized slip has already occurred at the periphery of the contact. Then, the interface crack propagates from the right to the left edge of the contact with a relatively sharp front. The location of this crack front as a function of time can be used to determine the average velocity of the interface crack which is found to be of the order of $100 \mathrm{~mm} \mathrm{~s}^{-1}$, i.e. four orders of magnitude higher that the driving velocity. Locally, the space derivative of the displacement field also provides an estimate of the in-plane shear strain of the PDMS substrate which is about $10 \%$. However, this value is probably underestimated due to the limitations in the spatial resolution of the displacement measurement $(100 \mu \mathrm{m}$ as fixed by the holes lattice). High strain and strain rates are thus probably experienced locally at the crack front. After full propagation of the crack across the contact, a relatively uniform displacement of about $70 \mu \mathrm{m}$ is eventually achieved within the contact, which corresponds to the caracteristic length obtained from the frequency/velocity relationship (see above). Figure 7 shows a similar analysis for stickslip motions at $1 \mathrm{~mm} \mathrm{~s}^{-1}$. The velocity fields and the displacement profiles presented in this figure now encompass a whole stick-slip period (i.e. $65 \mathrm{~ms}$ ). It appears that contact slip occurs in a slightly different way. Although there is still some evidence of a preferential crack nucleation at the lateral contact edge (here on the left), slip now propagates nearly homogeneously around the whole periphery of the contact. The last point to experience shear failure within the interface is now clearly located close to the center of the contact. The associated lateral load corresponds to the maximum of the stick slip cycle. It turns out that stick-slip motions at high velocities involve a pulsating slip annulus rather than the propagation of transverse cracks, as it is observed at low velocity. The average velocity of this slip annulus can estimated from the time changes in the location of the relatively sharp displacement front close to the boundary between the stick and slip zones (bottom part of Figure 7). The obtained value is about $120 \mathrm{~mm} \mathrm{~s}^{-1}$, i.e. a value very close to the velocity of slip pulses measured at $5 \mu \mathrm{m} \mathrm{s}^{-1}$. The velocity of the crack fronts associated with stick-slip motions thus remains remarkably unchanged when the driving velocity is increased by nearly four order of magnitudes.

\section{Discussion}

Stick-slip instabilities are often thought of as sharp jumps from a uniform stick state to a homogeneous sliding state. 

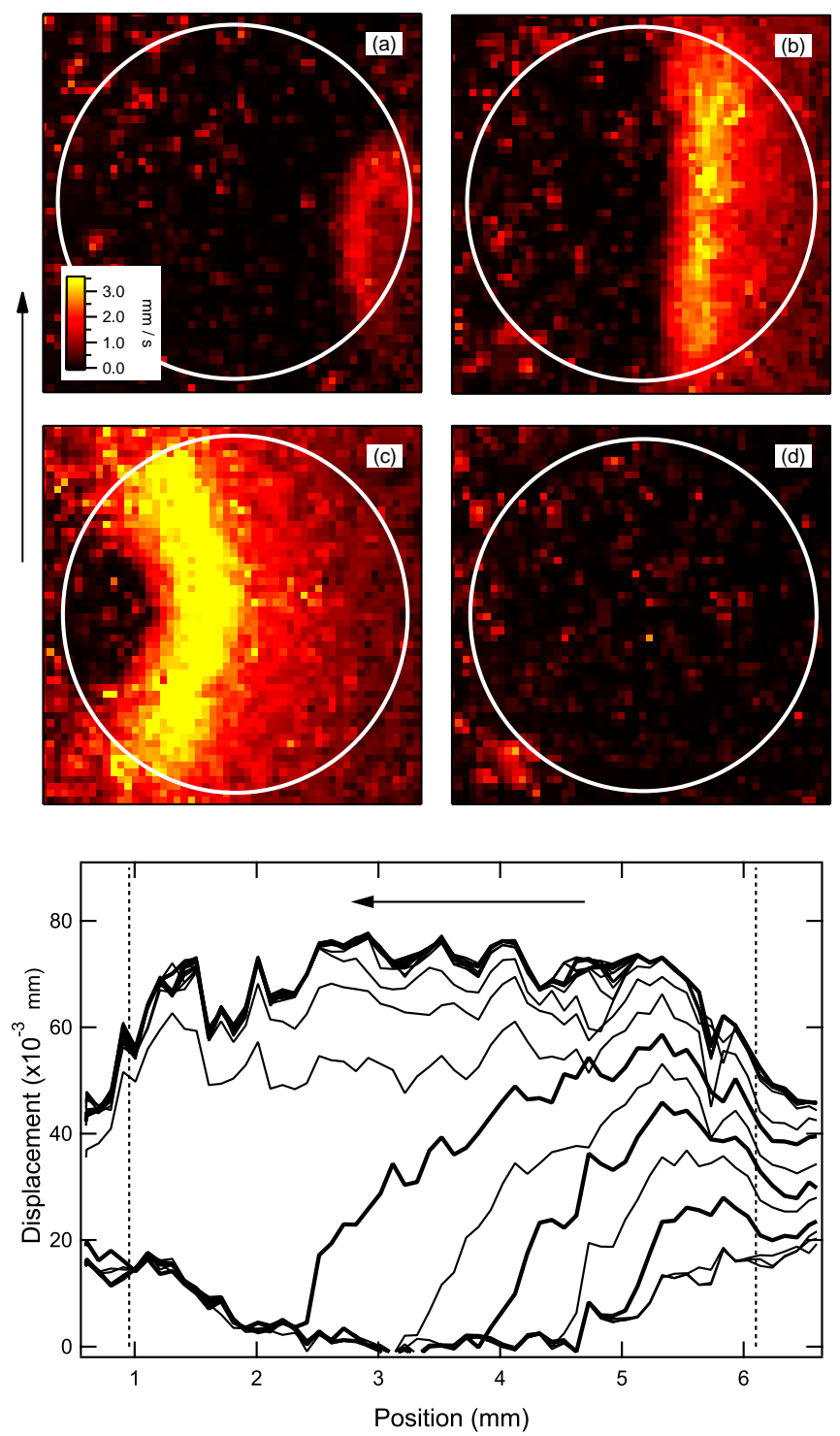

Fig. 6. Slip pulse propagation during a slip event at $5 \mu \mathrm{m} \mathrm{s}^{-1}$. Bottom: displacement profiles taken across the contact area and perpendicular to the sliding direction at $7.7 \mathrm{~ms}$ time intervals. The arrow indicates the direction of pulse propagation. Dotted lines delimits the contact edge. Top: sliding velocity fields at the contact interface. Pictures (a) to (d) corresponds to the displacement profiles in bold lines (from bottom to top). The measured sliding velocity corresponds to the time derivative of the surface displacement component along the sliding direction. As indicated by the vertical arrow, the PDMS substrate is moved from bottom to top with respect to the contact area which is delimited by a white circle. A slip pulse is propagating from the right to the left contact edge, perpendicularly to the direction of the imposed displacement. The contact radius is $2.5 \mathrm{~mm}$.
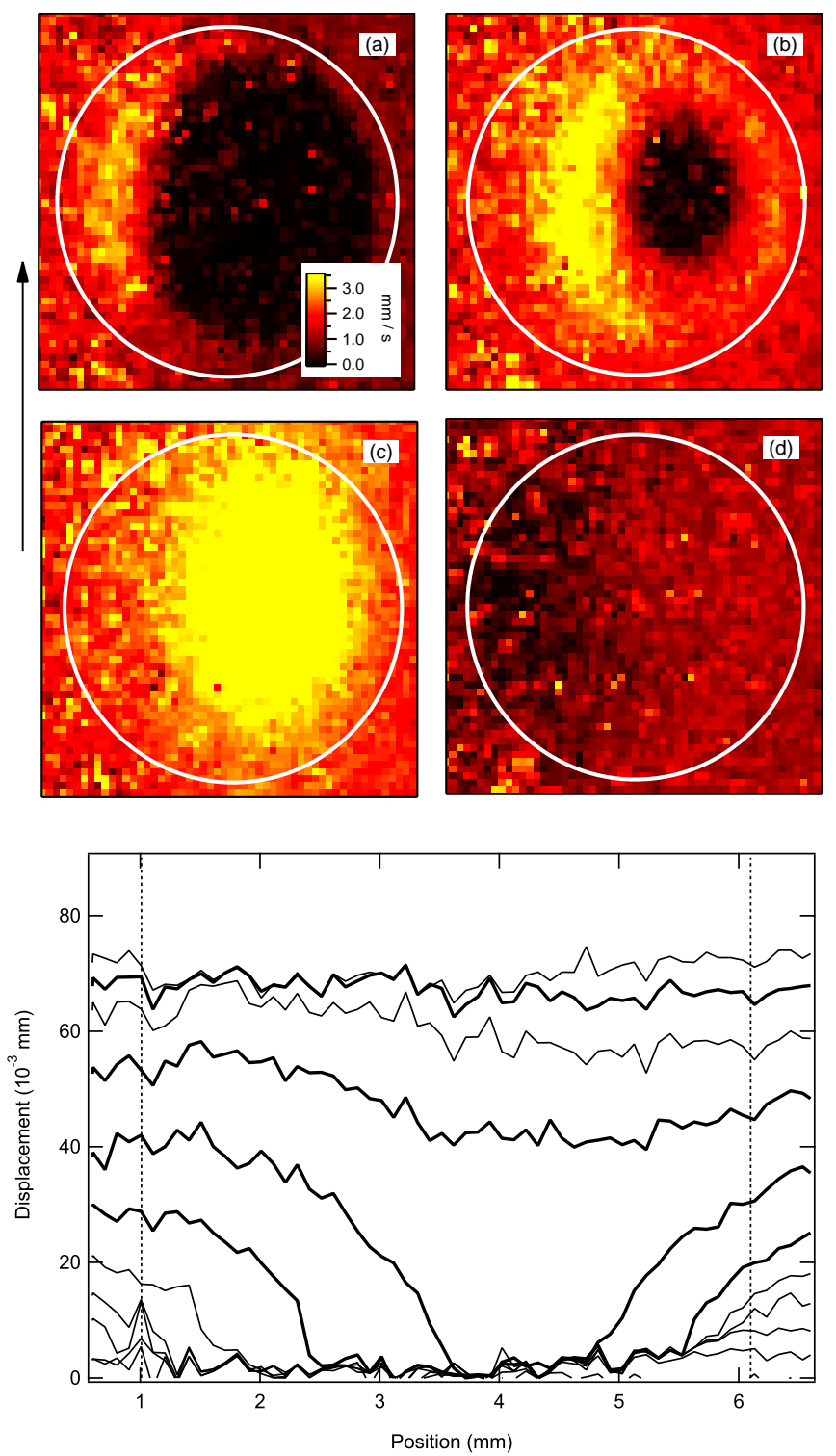

Fig. 7. Slip pulse propagation during stick-slip motions at $1 \mathrm{~mm} \mathrm{~s}^{-1}$. Bottom: displacement profiles taken across the contact area and perpendicular to the sliding direction at $7.7 \mathrm{~ms}$ time intervals during a stick-slip period. The dotted lines delimit the contact edge. Top: sliding velocity fields at the contact interface. Pictures (a) to (d) corresponds to the displacement profiles with bold lines (from bottom to top). The contact area is delimited by a white circle. The contact radius is $2.5 \mathrm{~mm}$.

The picture which emerges from our observations is quite different: the stick-slip motion results from a heterogeneous sliding process, with nucleation and propagation of interface cracks. These observations are reminiscent of earlier reports by Ronsin and co-workers [30,17,19]. They have studied the friction of planar gel/glass interfaces with a linear kinematics and found slip pulses associated with oscillations in the frictional shear stress below a critical driving velocity. Our discussion of the results is based on the comparison with theirs. 
Slip initiation occurs for comparatively constant tangential force. The force at the onset of the slip (the "static" friction threshold, even if in the face of the present evidence for heterogeneous dynamics its relevance becomes somewhat questionable) is quite constant over a range of velocities which spans three orders of magnitude (figure 2 ). Moreover, in our system, the cracks are invariably nucleated at the sides of the contact, but neither at the front nor the back. In comparison, in gel blocks, the cracks are nucleated at the back of the block. It is interesting to note that for our circular contact crack initiation actually occurs in mode III in agreement with the findings of Gao by FEM [9].

A second observation is that the velocity of the fracturelike slip fronts is almost independent of the driving velocity. This observation is again in agreement with previous results on gels [30]. However for our PDMS surfaces the crack front velocity is approximately $100 \mathrm{~mm} \mathrm{~s}^{-1}$ : this value is larger by one order of magnitude than the pulse velocity in the gel experiments $\left(8 \mathrm{~mm} \mathrm{~s}^{-1}\right)$ but it remains below the velocity of Rayleigh waves (about $10 \mathrm{~m} \mathrm{~s}^{-1}$ ). The propagation of the interface cracks across the contact is observed to result in an average Burgers vector (or slip displacement), $\delta_{\text {slip }} \approx 70 \mu \mathrm{m}$. This value is remarkably constant as the driving velocity is varied over orders of magnitude. The driving velocity $v$ is equal to $\delta_{\text {slip }} / T_{s s}$ where $T_{s s}$ is the stick-slip period. As a result the stickslip frequency $F=1 / T_{s s}$ must be proportional to $v$ as observed experimentally.

This view of friction as due to the motion of cracks raises a number of questions. From theoretical development, Ronsin et al [19] and Baumberger et al [17] have suggested that the pulse front velocity can be accounted for by the poro-elasticity of the gel which controls energy dissipation at the pulse tip via localized collective diffusion modes. We surmise that the crack velocity is dominated by viscous dissipation in the dislocation core, and the dissipation mechanisms are probably quite different [31]. Indeed in adhesive contact problems with rubbers, it is well known that $K_{c}$, or equivalently the fracture energy $G_{c}$, is rate dependent due to localized viscoelastic dissipation at the crack tip where high strain and strain rates are experienced [32, $32,31]$. In such a mode I experiment, it is indeed very common to find a three orders of magnitude enhancement of the fracture energy as the crack front velocity increases. We might expect traces of a similar behaviour in friction: it could be expected that the crack propagation velocity would depend upon the driving velocity, with resulting variations in the dissipated power. In fact this is not the case. The most striking feature in this stick-slip regime is that nothing depends upon the driving velocity except the stick-slip period, and even this occurs because of the trivial reasons that it allows the rest to stay constant. In order for the slip event to be independent from the driving velocity, the necessity which arises is that the threshold stress for nucleation is much higher than what would be needed for quasi static propagation, if it could be observed. Then when the crack has been initiated, there is a rather large amount of stored elastic energy which sets the crack moving through the contact at large velocity. Since the threshold is weakly sensitive to velocity (figures 2a and $2 \mathrm{~b}$ show an increase of about $30 \%$ between $5 \mu \mathrm{m} \mathrm{s}^{-1}$ and $1 \mathrm{~mm} \mathrm{~s}^{-1}$ ), the energy stored at crack initiation stays nearly unaffected and the crack dynamics is always the same. As a result both the velocity and the burgers vector are unchanged throughout the dynamic range. Therefore, it turns out that, in our system, the main effect of surface patterning is to weaken the interface which in turn promotes the nucleation and propagation of interface cracks at a reasonable value of stored elastic energy. This assumption is supported by the fact that the friction force with the smooth lens is about twice that of the patterned lens at a given velocity (i.e. $3.9 \mathrm{~N}$ and $4.2 \mathrm{~N}$ for $5 \mu \mathrm{m} \mathrm{s}^{-1}$ and $1 \mathrm{~mm} \mathrm{~s}^{-1}$, respectively). Larger initiation stresses would result in a different type of fracture which is described as uniform sliding. The control through the initiation stress explains why the typical length scales associated with the observed interface cracks is completely de-correlated from the characteristic sizes of the pattern. The question behind this characteristic slip displacement is to identify the parameters which control the manner in which the contacting surfaces re-stick after the propagation of the crack front. This complicated issue probably involve an intricate balance between the time and velocity dependence of the frictional stress and the release of the elastic energy stored in the system which would require further analysis.

The typical crack propagation time is $t_{p} \approx 2 a / v_{c}$ where $v_{c}$ is the crack velocity. At low velocity, $t_{p}$ is much lower than the stick-slip period. As a consequence, the contacting surfaces can re-stick to each other before a sufficient elastic energy is restored within the system to re-initiate a slip event. Conversely, crack propagation time at the highest velocities is close to the stick-slip period and much less time is left for the surfaces to re-stick. These considerations could qualitatively explain the transition from a saw tooth to a quasi-sinusoidal force signal when the driving velocity is increased. In brief, the stick-slip regime ends when the load for crack initiation is reached within the time needed for the crack to cross the contact zone.

\section{Conclusion}

In this study, we have shown that stick-slip motions within a contact interface between a rubber substrate and a rigid patterned glass lens involve crack-like events which are initiated at the lateral contact edges under a privileged mode III crack opening condition. Using the resources of in situ contact imaging, spatially resolved displacement fields were measured at the surface of the rubber specimen during the occurrence of slip events. One of the most striking feature in this stick-slip regime was that crack propagation occurs at a rate which remains remarkably independent on the driving velocity over four orders of magnitude. This was interpreted as evidence of a crack threshold which is much higher that what would be needed for quasistatic propagation. Here, the initiation stress is mainly controlled by the pattern on the glass surface which effect seems mainly to weaken the interface. This point would 
deserve further studies where, for example, the effects of pattern orientation, wavelength and amplitude would be considered.

\section{Acknowledgement}

Part of this work was supported by the National Research Agency (ANR) within the framework of the DYNALO project (project NT09 499845). We also acknowledge financial support from Saint Gobain Recherche, Aubervilliers, France.

\section{References}

1. M. Bengisu, A. Akay, Journal of Acoustical Society of America 105, 194 (1999)

2. A. Baldassari, F. Dalton, A. Petri, S. Zapperi, G. Pontuale, L. Pietronero, Physical Review Letters 96, 118002 (2006)

3. S. Nasuno, A. Kudrolli, A. Bak, J. Gollub, Physical Review E 58, $2161(1998)$

4. J. Woodhouse, Acustica 80, 58 (1994)

5. J. Rice, A. Ruina, Journal of Applied Mechanics 50, 343 (1983)

6. A. Ruina, Journal of Geophysical Research 88, 359 (1983)

7. E. Brener, S. Malinin, V. Marchenko, European Physical Journal E 17, 101 (2005)

8. C. Caroli, Physical Review E 62, 1729 (2000)

9. Y. Gao, Journal of the Mechanics and Physics of Solids 58, 2023 (2010)

10. E. Gerde, M. Marder, Nature 413, 285 (2001)

11. O. Ben-David, G. Cohen, J. Fineberg, Science 330, 211 (2010)

12. S. Rubinstein, G. Cohen, J. Fineberg, Journal of Physics D: Applied Physics 42, 214016 (2009)

13. S. Rubinstein, G. Cohen, J. Fineberg, Physical Review Letters 98, 226103 (2007)

14. S.M. Rubinstein, M. Shay, G. Cohen, J. Fineberg, International Journal of Fracture 140(1-4), 201 (2006)

15. R. Progri, B. Villechaise, Comptes Rendus de l'Académie des Sciences Série II 299, 763 (1984)

16. M.A. Rubio, J. Galeano, Physical Review E 50, 1000 (1994)

17. T. Baumberger, C. Caroli, O. Ronsin, European Physical Journal E 11, 85 (2003)

18. T. Baumberger, C. Caroli, O. Ronsin, Physical Review Letters 88, 075509 (2002)

19. O. Ronsin, T. Baumberger, C. Hui, The Journal of Adhesion 87, 504 (2011)

20. A. Schallamach, Wear 17(4), 301 (1971)

21. A. Chateauminois, C. Fretigny, European Journal of Physics E 27, 221 (2008)

22. A. Chateauminois, C. Fretigny, L. Olanier, Physical Review E 81, 026106 (2010)

23. D. Nguyen, P. Paolino, M.C. Audry, A. Chateauminois, C. Frtigny, Y.L. Chenadec, M. Portigliatti, E. Barthel, Journal of Adhesion 87, 235 (2011)

24. E. Gacoin, C. Fretigny, A. Chateauminois, Tribology Letters 21, 245 (2006)

25. A. Letailleur, J. Teisseire, N. Chemin, E. Barthel, E. Sondergard, Chemistry of Materials 22, 3143 (2010)
26. R. Mindlin, ASME Transactions, Journal of Applied Mechanics, Series E 16, 327 (1953)

27. M. Barquins, D. Maugis, R. Courtel, Comptes Rendus Hebdomadaires des Scéances de l'Académie des Sciences Série B 280(4), 49 (1975)

28. K. Johnson, Proceedings of the Royal Society A 453, 163 (1997)

29. A. Savkoor, G. Briggs, Proceedings of the Royal Society of London Series A 356, 103 (1977)

30. T. Baumberger, C. Caroli, O. Ronsin, Physical Review Letters 88, 075509 (2002)

31. E. Barthel, C. Fretigny, Journal of Physics D: Applied Physics 42, 195302 (2009)

32. D. Maugis, M. Barquins, Journal of Physics D: Applied Physics 11, 1989 (1978) 